\title{
Anormalidades electroencefalográficas en pacientes pediátricos hondureños con desnutrición proteico-energética: un estudio de casos y controles
}

Miguel Solano*, Marco Tulio Medina**, Rebeca Hernández**, José Lara

\section{RESUMEN}

Se tienen como objetivos determinar y caracterizar los hallazgos electroencefalográficos en pacientes pediátricos con desnutrición proteico energética, utilizando un estudio de casos y controles.

Se diseñó un estudio analítico-descriptivo con un grupo de pacientes y un grupo de controles sanos. Se realizó en el período comprendido de Febrero 2007 hasta Junio 2008.Se recolectaron los pacientes en una escuela localizada en un población marginal de Comayagüela. Se captaron 40 pacientes con desnutrición e igual número de controles sanos. Se realizó una evaluación antropométrica, neurológica y laboratorial. Se efectuaron registros de EEG digital en ambos grupos, siendo evaluados en forma ciega por dos médicos neurofisiólogos clínicos.

Un 42\% de los pacientes con desnutrición protéico- energética mostraron actividades electroencefalográficas anormales ( $P=0.0000045)$ : a) Actividad epileptiforme focal en un $20 \%$, b) Actividad lenta focal en un $15 \%$, c) Actividad epileptiforme generalizada en un $12.5 \%$, d) Actividad lenta generalizada en un $10 \%$. Los grupos etarios más afectados fueron los que comprendían las edades entre 69 años.

Se encontraron frecuentes alteraciones en el trazado electroencefalográfico de los pacientes con desnutrición protéico-energética; alcanzando un $42 \%$ en comparación al grupo de controles sanos.

Palabras clave: Electroencefalograma, desnutrición, electrofisiología, nutrición, trastornos neurológicos, enfermedades carenciales

* Universidad Nacional Autónoma de Honduras. Postgrado de Neurología. Medico residente

* Universidad Nacional Autónoma de Honduras. Profesores de Postgrado de Neurología. Hospital Escuela

${ }^{\dagger}$ Epidemiólogo, y estadistico 


\section{ABSTRACT}

To determine and characterize the electroencephalographic findings of pediatric patients with protein-energy malnutrition using a case-control study.

An analytic descriptive study was developed with a group of patients and a healthy control group during the period of February 2007, through June 2008. The forty patients were gathered from a low-class school located in Comayagüela, matching their number to the healthy control group. Each patient went through an anthropometric, neurological, and laboratorial evaluation. Digital electroencephalography (EEG) was performed to both groups, which was later blindly evaluated by two clinician neurophysiologists.

$42 \%$ percent $(P=0.0000045)$ of the malnourished patients showed the following abnormal EEG tracks: a) focal discharge activity in $20 \%$, b) slow discharge activity in $15 \%$, c) global discharge activity in $12.5 \%$, and d) slow global discharges in $10 \%$. The most affected ages were between 6 and 9 years old.

A high frequency of alterations on EEG tracks was found on patients with proteinenergy malnutrition, reaching a $42 \%$ compared to a healthy control group.

Key words: Electroencephalography, protein-energy malnutrition, electrophysiology, nutrition, neurological disorders, deficiency disease. 


\section{INTRODUCCIÓN}

La Organización Mundial de la Salud reportó en el 2007 que 1000 millones de personas padecen de trastornos neurológicos, lo que causa una importante morbilidad y mortalidad a nivel mundial, con un $12 \%$ de las muertes anuales. Los trastornos asociados a la desnutrición con frecuencia producen morbilidad y discapacidad importante. La desnutrición puede producir varios tipos de alteraciones neurológicas, tanto a nivel periférico como a nivel del sistema nervioso central, 0 afectando ambos simultáneamente ${ }^{(1-3)}$.

La magnitud del problema es importante sobre todo en regiones como Latinoamérica, Asia y África en donde se estima que hay al menos 192 millones de niños con desnutrición. En donde el 31\% de los niños tiene bajo peso y el 38\% retardo del crecimiento relacionado hasta en un 53\% con la muerte asociada a infecciones respiratorias, muerte perinatal, malaria, diarrea, etc. Por lo que se considera un problema de salud pública ${ }^{(1-3)}$.

En Honduras el $71 \%$ de la población se encuentra en la línea de pobreza y un 24\% vive con menos de un dólar diario. En el año 2001 la desnutrición afectaba a más del $40 \%$ de los niños y más del $32 \%$ de las niñas ${ }^{(3)}$.

En nuestro medio, a pesar de su alta incidencia, existen escasos estudios sobre este problema. Debido a lo anterior sería importante determinar y caracterizar los hallazgos electroencefalográficos en pacientes con desnutrición, comparados con controles sanos.

\section{METODOLOGÍA}

Diseño metodológico y período de estudio. Se diseñó un estudio analíticodescriptivo con un grupo de pacientes con desnutrición proteico-energética (DPE) y un grupo de controles sanos. Se realizó en el período comprendido de Febrero 2007 hasta Junio 2008 (18 meses). Los hallazgos electroencefalográficos encontrados se analizaron según su estado nutricional, edad y sexo. Previo a su inicio el proyecto se socializó en la escuela donde se desarrolló el trabajo.

Para la recolección de datos se utilizó una encuesta donde se valoraban las mediciones antropométricas, la historia clínica neurológica, antecedentes, exámenes laboratoriales: hemograma, glucosa, BUN, creatinina, sodio (Na), potasio (K), transaminasas (TGO, TGP), y la descripción de los hallazgos electroencefalográficos. 
Lugar de estudio. Se escogió la escuela 15 de Septiembre del barrio Carrizal de Comayagüela, ubicada en un área urbano-marginal. El total de alumnos matriculados en el 2008 fue de 250. Los niños de la escuela estaban dentro de un programa de higiene dental, y eran evaluados periódicamente. El área de influencia es de aproximadamente 150 mil personas.

Muestra poblacional. El universo del estudio fueron todos los alumnos de la escuela, que comprendían edades entre 5-15 años. Los criterios de exclusión incluyeron los siguientes:

- Niños con antecedentes patológicos como epilepsia, traumatismo cráneoencefálico, neuroinfección, antecedentes familiares de epilepsia, antecedentes patológicos en el nacimiento (asfixia neonatal, uso de fórceps, etc.).

- Niños con alteraciones en el hemograma como anemia severa ( $\mathrm{Hb}$ menor de $8 \mathrm{mg} / \mathrm{dl}$ ), alteraciones en pruebas renales (BUN, creatinina), alteraciones hepáticas (transaminasas), alteraciones electrolíticas.

Aspectos bioéticos. Previa explicación, a cada padre/madre del paciente 0 responsable de él, se solicitó su consentimiento informado para participar voluntariamente en el estudio; siguiendo las normas internacionales establecidas.

Se les aclaró que al rechazar la participación en el estudio, en cualquier momento del desarrollo del mismo, quedarían excluidos inmediatamente sin comprometer la calidad de atención médica. El equipo investigador aseguró una estricta confidencialidad de la información de cada paciente hasta el final del estudio.

Recolección de datos. Bajo consentimiento informado de los padres, se realizó una primera fase en donde se caracterizó al paciente según su edad, sexo y posteriormente una evaluación antropométrica del peso, talla y perímetro cefálico, así mismo una historia con examen neurológico.

De acuerdo a las evaluaciones se determinó el tipo de desnutrición y el grado de severidad. Para ello se utilizó la escala de clasificación de Waterlow, la que divide a los pacientes en desnutrición DPE aguda (peso/talla) leve, moderada o severa; DPE crónica (talla/edad) leve, moderada o severa y DPE crónica agudizada. ${ }^{(4,5)}$ ver cuadro 1. 
Cuadro 1: Intensidad de la desnutrición e hipo crecimiento

\begin{tabular}{|l|c|c|}
\multicolumn{1}{c|}{$\begin{array}{c}\text { Grado de } \\
\text { Desnutrición }\end{array}$} & $\begin{array}{c}\text { Talla para } \\
\text { la edad }\end{array}$ & $\begin{array}{c}\text { Peso para } \\
\text { la talla }\end{array}$ \\
\hline 0, normal & Mayor 95 & Mayor 90 \\
\hline 1, leve & $90-95$ & $81-90$ \\
\hline 2, moderado & $85-89$ & $70-80$ \\
\hline 3, intenso & Menor 85 & Menor 70 \\
\hline
\end{tabular}

En una segunda fase se realizaron exámenes de hemograma, TGO y TGP, pruebas renales y electrolitos; con el objetivo de determinar si existe una condición que pueda producir alteraciones en el examen electroencefalográfico.

En la tercera fase se hizo, en aquellos pacientes que tenían sus evaluaciones normales, un estudio de electroencefalograma digital.

\section{Evaluación electroencefalográfica}

\section{DESCRIPCIÓN TÉCNICA}

Se practicó a los controles y casos un estudio electroencefalográfico digital de 30 minutos de duración, utilizando un equipo Cadwell computarizado de 32 canales. Se colocaran 19 electrodos en el cuero cabelludo según el Sistema Internacional Jasper de colocación de electrodos 10/20 y electrodos en el tórax para monitoreo electrocardiográfico. Se realizó la apertura y cierre de párpados durante un minuto, así como las maniobras de hiperventilación durante 3 minutos y fotoestimulación con estímulos luminosos intermitentes de frecuencia creciente entre 1 y $20 \mathrm{~Hz}$.

Operacionalización de variables. Se emplearon las definiciones de desnutrición de la OMS y de textos de pediatría, así mismo la clasificación de desnutrición de Waterlow. ${ }^{(4)}$ La definición de hallazgos electroencefalográficos fue dada por los textos de electroencefalografía y neurofisiología e interpretados por médicos especialistas en neurofisiología clínica.

Análisis estadísticos. Inicialmente se efectuó un análisis de las variables cuantitativas y cualitativas; comparando los resultados del grupo de trabajo con el grupo de comparación. Se calcularon las medias, frecuencias y valores P en función de determinar los resultados normales y tendencias de las variables, basadas en la edad, sexo, hallazgos en el EEG. 
La matriz de datos fue construida en el paquete de Microsoft Office Excel 2003 y el análisis estadístico se realizó con el paquete Epi-Info versión 3.5.1. A la vez se estableció una significancia estadística para valores de $p<0.05$.

\section{RESULTADOS}

Descripción de la población. Durante el período de estudio se investigó un total de 80 niños y niñas, divididos en 2 grupos, uno con desnutrición proteico-energética (DPE), $n=40$ y otro de controles sanos, $n=40$. En ambos grupos había igual cantidad de niños $(n=22)$ y niñas $(n=18)$. No se encontró diferencia significativa en cuanto al sexo $(P=0.588)$ ni tampoco en cuanto a las medias de edades entre ambos grupos $(P=0.80)$. Así mismo, se encontró que el único parámetro laboratorial con diferencia significativa fue la concentración de hemoglobina $(\mathrm{P}=0.00002)$, siendo el promedio de 12,12 gr/dl para los controles sanos vrs $11.0 \mathrm{gr} / \mathrm{dl}$ para el grupo de pacientes con desnutrición.

En el grupo con DPE 18 eran de sexo femenino y 22 masculinos al igual que en el grupo de pacientes sin DPE. Las edades estuvieron comprendidas entre los 6-12 años, concentrándose más en los grupos de 6 y 7 años $n=41(50 \%)$, seguido por el grupo de 8-9 años con un $n=20,(25 \%)$.

Estado nutricional y tipo de DPE. La evaluación del estado nutricional se estableció con medidas antropométricas y se clasificó según la escala de Waterlow Para el grupo de casos encontramos que la DPE más frecuente fue la aguda leve, $n=18$ (45\%); seguida de las formas moderadas tanto en su forma aguda como crónica ( $n=6),(15 \%)$ y $n=8,(20 \%)$. Respectivamente, el grupo de pacientes con desnutrición crónico agudizada fue de $n=7$ (17.5\%) y uno para la desnutrición aguda grave (2.5\%).

La distribución del tipo DPE en cuanto al sexo, no demostró mayor diferencia. DPE aguda leve hubo mayor porcentaje en el sexo femenino $n=11$ (61\%) contra el masculino $n=7$ (39\%). En la DPE aguda moderada los varones mostraron mayor frecuencia $n=5(100 \%)$ contra ningún caso en el sexo femenino.

EEG. Se realizaron 80 registros electroencefalográficos. En donde se estudiaron las siguientes características: frecuencia, amplitud, reactividad, cambios con las maniobras de activación y descripción de las anormalidades.

Frecuencias de EEG y DPE. Al determinar las medias de las frecuencias que obtuvimos en los trazados electroencefalográficos, encontramos diferencias entre 
los dos grupos. Se observa que los controles sanos tienen actividad de fondo con frecuencias medias superiores a los del grupo de pacientes con DPE. Así mismo, si dividimos el grupo de pacientes con DPE en: DPE con EEG normales y DPE con EEG anormales, el primer grupo tiene medias mayores que el segundo. Los controles sanos mostraron un patrón lineal con frecuencias superiores a los 8 ciclos por segundo, en cambio, los pacientes con DPE y EEG anormal tenían medias de frecuencias por debajo de 7.5 .

Amplitudes EEG y DPE. Se encontraron diferencias entre las amplitudes de los grupos ya mencionados; siendo los controles sanos los que tienen amplitudes mayores, y el grupo de pacientes con DPE amplitudes menores. Se debe destacar que ambos grupos tenían amplitudes de rangos normales. Los valores medios de amplitudes para el grupo de controles sanos fueron por arriba de los 70 microvoltios, en cambio los pacientes con DPE y EEG anormales mostraron valores menores a los 60 microvoltios.

Gradiente antero posterior y DPE. El gradiente antero-posterior se caracteriza por presentar una mayor amplitud en regiones posteriores con una actividad de fondo normal para la edad y menores amplitudes en regiones anteriores con actividades más rápidas. Al evaluar dicho gradiente en el grupo de pacientes con DPE encontramos que en 4 de 40 no estaba adecuado, siendo 3 de los casos del grupo perteneciente a pacientes con DPE aguda. En cambio, en el grupo de controles sanos no se encontraron alteraciones en el gradiente.

Hallazgo EEG en DPE. Dividimos los hallazgos en normales y anormales. En el primer grupo se enumeran variantes fisiológicas en ambas poblaciones de pacientes, encontrando EEG normales o dentro de límites normales en un $100 \%$ de los controles sanos y un $57.5 \%$ ( $n=23$ ) en el grupo de pacientes con DPE, con $P=0.0000045$. Ver tabla 1.

Se encontró que las anormalidades en el electroencefalograma para la DPE aguda leve mostró mayor frecuencia de hallazgos anormales en ocho pacientes; siendo estas de tipo epileptiforme focal en un paciente, epileptiforme generalizado en dos, actividad lenta focal en dos y lenta generalizada en tres pacientes. DPE aguda moderada registró anormalidades en un 66\% de tipo epileptiforme focal en tres pacientes y uno en actividad epileptiforme generalizada. La DPE crónica moderada presentó anormalidades en tres pacientes; siendo un paciente en cada tipo: actividad epileptiforme focal, lenta focal y lenta generalizada. Ver tabla 2. 
Tabla 1. Porcentaje y frecuencia de EEG normal o anormal, en pacientes con desnutrición y controles sanos

\begin{tabular}{|c|c|c|c|}
\hline DPE & $\begin{array}{c}\text { EEG } \\
\text { anormal (N) }\end{array}$ & $\begin{array}{c}\text { EEG } \\
\text { anormal (N) }\end{array}$ & Total (N/\%) \\
\hline Si & $17 / 42.5 \%$ & $23 / 57.5 \%$ & $40 / 100 \%$ \\
\hline No & 0 & $40 / 100 \%$ & $40 / 100 \%$ \\
\hline
\end{tabular}

DPE= desnutrición proteico energética, $E E G=$ electroencefalograma

Se observa que $17 / 40$ es decir un $42 \%$ de los pacientes con DPE presentaban EEG anormal. $P=0.0000045$

Tabla 2. Distribución de frecuencias de los EEG normales o anormales en el grupo de pacientes con desnutrición según su tipo

\begin{tabular}{|c|c|c|c|c|c|c|}
\hline EEG & $\begin{array}{c}\text { DPEA } \\
\text { grave } \\
(\mathbf{N})\end{array}$ & $\begin{array}{c}\text { DPEA } \\
\text { Moderada } \\
(\mathbf{N})\end{array}$ & $\begin{array}{c}\text { DPEA } \\
\text { Leve } \\
(\mathbf{N})\end{array}$ & $\begin{array}{c}\text { DPEA } \\
\text { Moderada } \\
(\mathbf{N})\end{array}$ & $\begin{array}{c}\text { DPECA } \\
(\mathbf{N})\end{array}$ & $\begin{array}{c}\text { Total } \\
(\mathbf{N} / \%)\end{array}$ \\
\hline Anormal & 1 & 4 & 8 & 3 & 1 & $17 / 42.5 \%$ \\
\hline Normal & 0 & 2 & 10 & 5 & 6 & $23 / 57.5 \%$ \\
\hline
\end{tabular}

DPE = desnutrición proteico energética, EEG= electroencefalograma, DPECA= desnutrición proteico-energética crónica agudizada DPEA = desnutrición proteico-energética aguda y DPEC = desnutrición proteico-energética crónicsa

Muestra la distribución de frecuencias según estado nutricional y si el EEG es normal o no, encontrando que en la DPE aguda leve la relación de EEG anormal es de $8 / 18$, DPEA moderada es de 4/6, la DPEC Moderada es de $3 / 8$ y en la DPE crónica agudizada apenas es de 1/7. Con una $P=0.0000045$.

Las actividades que se encontraron fueron de diferentes tipos: actividad epileptiforme focal y/o generalizada, actividad lenta focal y/o generalizada. En nuestra serie de casos, los pacientes con actividad epileptiforme focal fueron 8; distribuyéndose a nivel frontal en 3 casos, occipital en 2 y el restante en diversas áreas. 
La actividad lenta focal se observó en 6 casos, a nivel frontal en 3 ocasiones y los restantes en otras áreas. Se observó que en su mayoría las anormalidades encontradas se deben a enlentecimiento de forma generalizada, actividad epileptiforme generalizada y actividad epileptiforme focal. No hay predisposición por el sexo.

También se cruzó por grupos de edad, si el EEG era normal o anormal, encontrándose que la mayoría de los pacientes con EEG anormales se establecían en los grupos de menor edad; con un $29.3 \%$ en el grupo de $6-7$ años y un $21.1 \%$ en el de 8-9 años, con una $P=0.143$.

Otro de los hallazgos en el EEG fue la actividad epileptiforme focal, la misma se presentó en cuatro pacientes con DPE aguda leve, tres en DPE aguda moderada y uno en DPE crónica moderada, con una $\mathrm{P}=0.0026$. Ver figura 1 de ejemplo y gráfico 1 para distribución de frecuencia.

Figura 1. Actividad epileptiforme focal

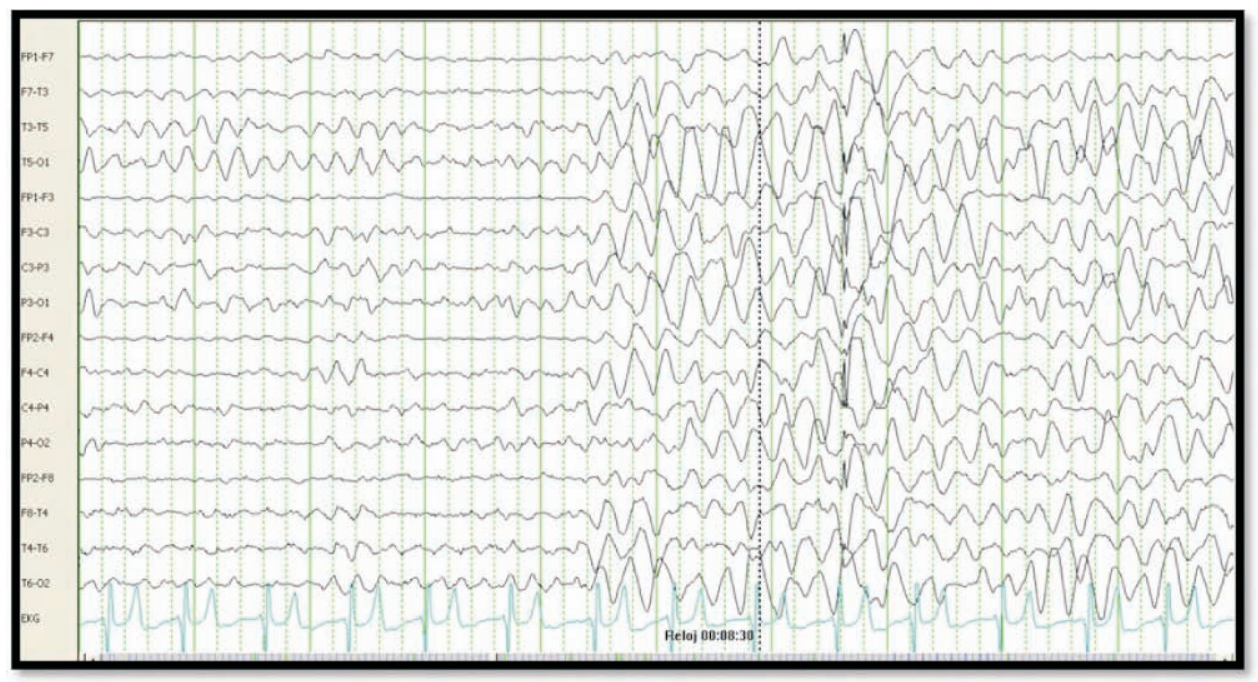

El EEG es un ejemplo de actividad epileptiforme focal de tipo punta onda en los electrodos FP1, F7, F3, C3, FP2, F8, F4, C4. 
Gráfico 1. Frecuencias de pacientes con DPE y actividad epileptiforme focal

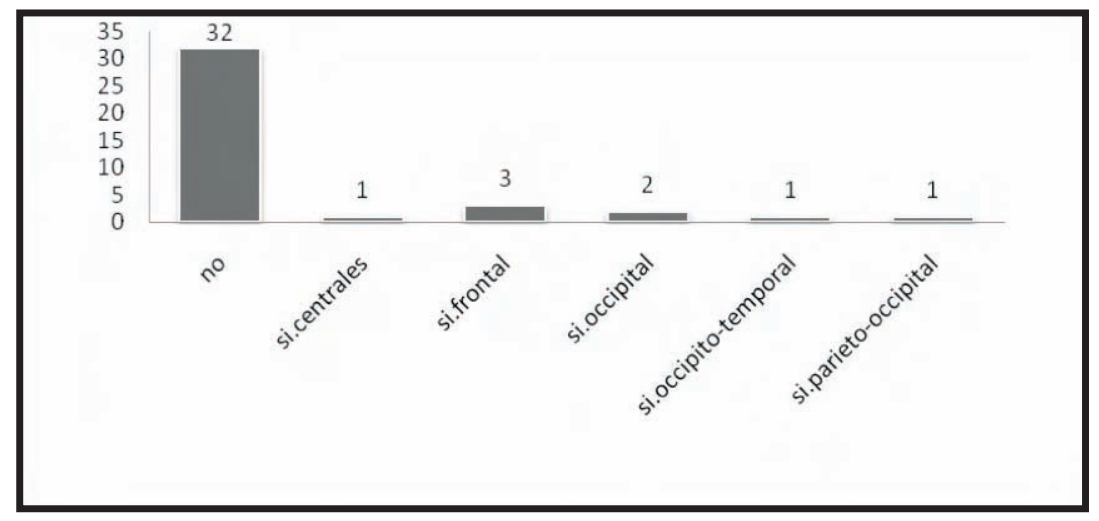

En este gráfico observamos los diferentes tipos de actividad epileptiforme focal. En el eje de las $X$ se describe el tipo de actividad. En el eje de las $Y$ se enumera la frecuencia. El porcentaje de anormalidades representa un $20 \%(n=8)$ con una $\mathrm{P}=0.1136$

La actividad epileptiforme generalizada se reportó en cinco pacientes con DPE. Con una $P=0.0273$. Así mismo, al evaluar las frecuencias de APG, en cuanto al sexo, encontramos que dos de los casos se dieron en el sexo femenino y tres en el sexo masculino. También se observó que dicho tipo de hallazgo se presenta más en los grupos de edades inferiores; siendo el grupo de 8-9 años el más afectado con tres pacientes del total, seguido por el grupo de 6-7 años con 2 pacientes. Los grupos de mayor edad no presentaron este tipo de hallazgo. El siguiente es ejemplo (figura 2) de actividad epileptiforme generalizada de tipo punta onda.

Figura 2. Actividad epileptiforme generalizada

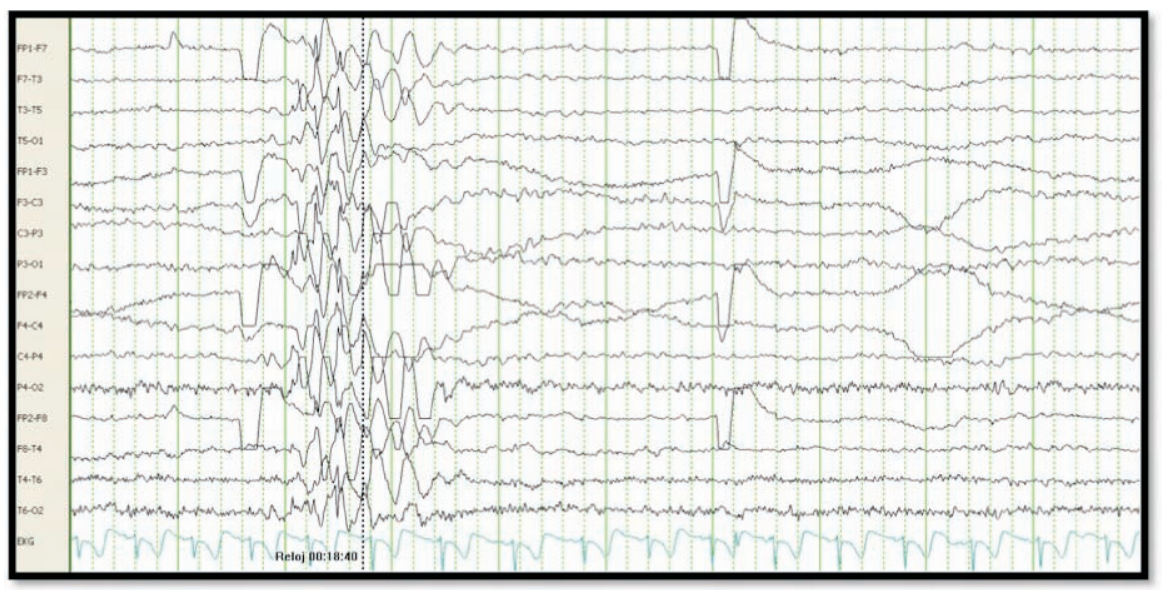


La flecha negra señala el grafo elemento de tipo punta onda generalizada de 2-3 ciclos por segundo. Otro hallazgo frecuente fue la actividad lenta focal, encontrándose en un 15\% del total de pacientes con DPE. Ésta fue variada en cuanto al foco, encontrándose a nivel frontal en un 7.5\%, otras regiones como la parieto-occipital, centro-parietal y occipital con un $2.5 \%, P=0.0127$. La distribución por sexo de dicho hallazgo es superior en el sexo femenino con un $11.1 \%$ vs un $4.5 \%$ del sexo masculino. La distribución por grupos de edades es variada; un 19\% en el grupo de 6-7 años, $12.5 \%$ en el grupo de 8-9 años y 25\% en el grupo de 12-13 años. El grupo de 10-11 años no mostró casos con este tipo de hallazgos. Ver figura 3 como ejemplo de actividad lenta focal.

La actividad lenta generalizada. Como hallazgo se encontró en un $10 \%(n=4)$ con $\mathrm{P}=0.0501$ en el grupo de pacientes con DPE. Su distribución por grupos de edades se demostró en dos pacientes; en el grupo de 12-13 años, uno en el de 8-9 años y uno en el de 6-7 años. El grupo de 10-11 años no reportó hallazgos. Por sexo, la actividad lenta generalizada se mostró, similar con un $11 \%$ en mujeres vs un $9 \%$ en los hombres.

La actividad lenta generalizada fue el hallazgo menos frecuente en esta serie. Ver figura 4 como ejemplo. Todos los datos se sometieron a prueba de Fisher para obtener la probabilidad. Ver tabla 4.

Figura 3. Actividad lenta focal

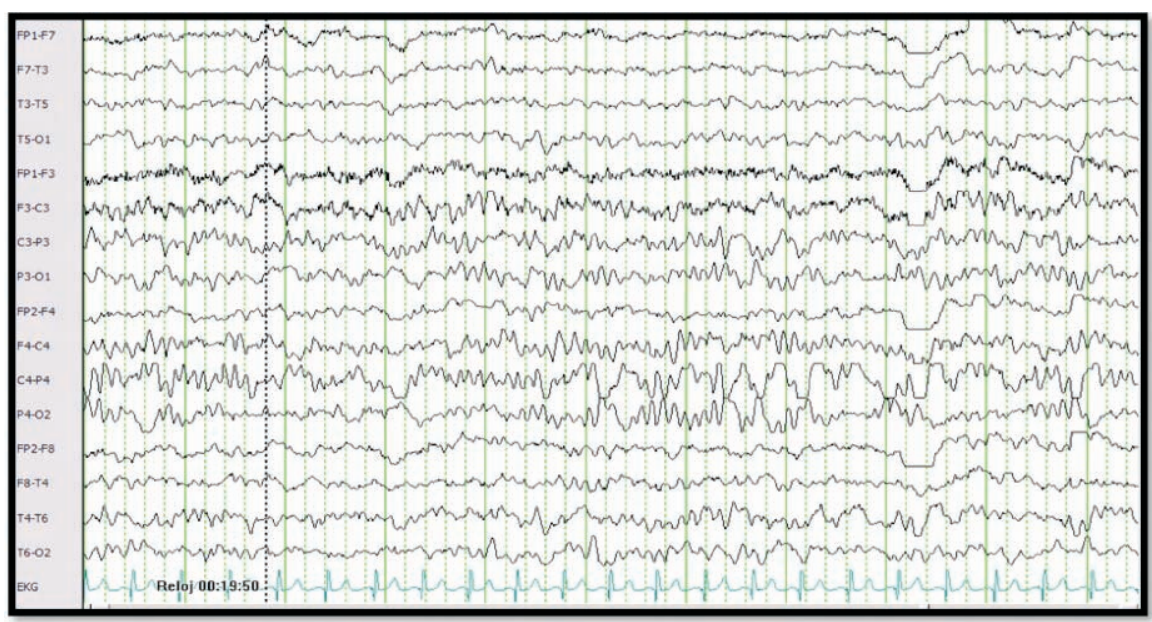

Se observan ondas lentas en electrodos C4-P4 acompañados de actividad irritativa de tipo punta en oposición de fase. 
Figura 4. Actividad lenta generalizada

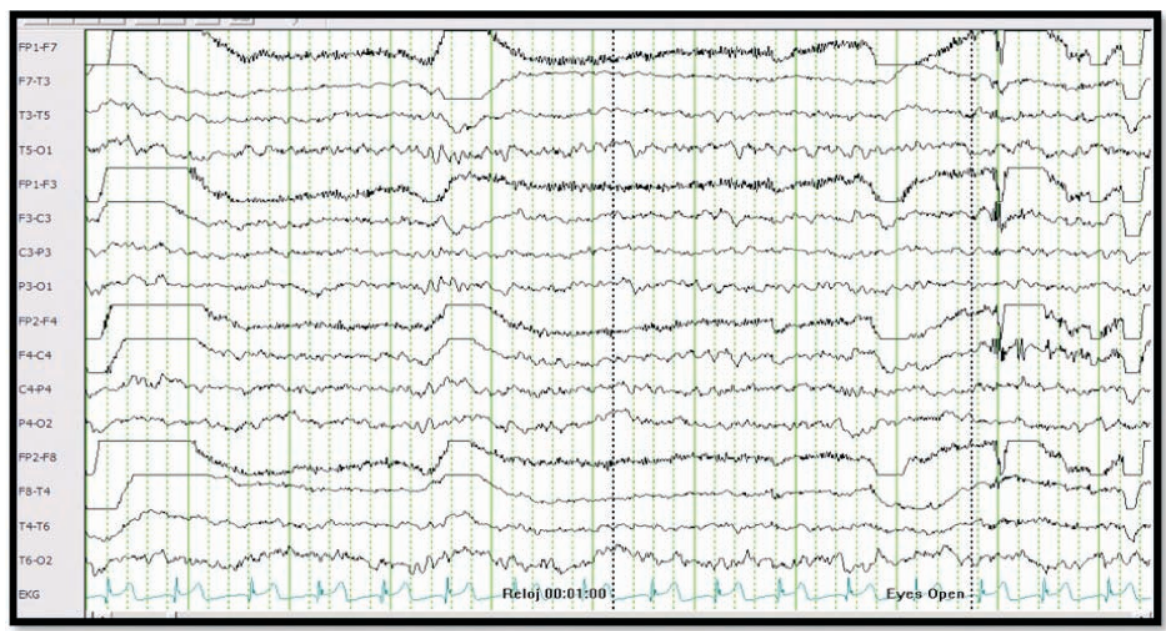

Las flechas muestran la actividad con ondas lentas (Theta) que están de forma generalizada.

Tabla 4. Comparación de los principales hallazgos y características entre los pacientes y grupo control que fueron evaluados

\begin{tabular}{|c|c|c|c|c|}
\hline Aspecto y Hallazgo & Normal & DPE & $\begin{array}{l}\text { Desv. } \\
\text { estandar }\end{array}$ & Probabilidad \\
\hline Actividad fondo: frecuencia & 9.125 & 8.675 & 0.7986 & 0.0921 \\
\hline Actividad de fondo: amplitud & 68.37 & 59.3 & 1.4626 & 0.0123 * \\
\hline $\begin{array}{l}\text { Reactividad EEG anormal } \\
(\mathrm{N})\end{array}$ & Ninguno & $4 / 40$ & - & 0.0501 \\
\hline $\begin{array}{l}\text { Gradiente antero-posterior } \\
\text { anormal (N) }\end{array}$ & Ninguno & $4 / 40$ & - & 0.0501 \\
\hline $\begin{array}{l}\text { Actividad lenta generalizada } \\
(\mathrm{N})\end{array}$ & Ninguno & $4 / 40$ & - & 0.0501 \\
\hline Actividad lenta focal (N) & Ninguno & $6 / 40$ & - & 0.0127 * \\
\hline $\begin{array}{l}\text { Actividad epileptiforme } \\
\text { generalizada (N) }\end{array}$ & Ninguno & $5 / 40$ & - & 0.0273 * \\
\hline $\begin{array}{l}\text { Actividad epileptiforme focal } \\
(\mathrm{N})\end{array}$ & Ninguno & $8 / 40$ & - & 0.0026 \\
\hline EEG normal (N) & 40 & 63 & - & 0.0000045 * \\
\hline
\end{tabular}

La probabilidad es estadísticamente significativa $\left(^{*}\right)$ cuando $\mathrm{P}$ es menor 0 igual a 0.05, según prueba de Fisher. 


\section{DISCUSIÓN}

Este es el primer estudio hondureño en el cual se comparan los hallazgos de electroencefalografía en pacientes con desnutrición y controles sanos.

De acuerdo a lo encontrado, podemos decir que hemos establecido una relación entre los hallazgos anormales y la desnutrición proteico-energética. Dicho hallazgo ya se había reportado anteriormente en la literatura mundial. Es conocido que la desnutrición en etapas tempranas produce retardo en la maduración cerebral; manifestando cambios en el ritmo del electroencefalograma ${ }^{(1)}$. Los parámetros como: las frecuencias, amplitudes, reactividad, adecuado gradiente u organización antero-posterior fueron claramente menores o anormales en el grupo de pacientes con desnutrición. Así mismo, las alteraciones patológicas también se mostraron en el grupo con desnutrición.

Diferentes autores ya habían reportado anormalidades electroencefalográficas; evidenciando las actividades lentas focales o generalizadas como más frecuentes y en menor cuantía las paroxísticas ${ }^{(1-7)}$. En la desnutrición aguda proteica 0 Kwashiorkor, se ha encontrado actividad de fondo lenta con grados de apatía mental; así mismo, actividades lentas temporales ${ }^{(8)}$.

Nosotros encontramos mayor número de alteraciones de tipo epileptiformes; llegando a ser un 20\% del tipo focal y $12.5 \%$ generalizada. Ésto podría explicarse debido a que la mayoría de los pacientes que manifestaron desnutrición estaban dentro de las edades escolares 6-7 años, edades en las que todavía se producen cambios en la maduración cerebral. Al haber carencia de micronutrientes se produce mayor susceptibilidad a dichas alteraciones. Sin embargo, debe mencionarse que dichas alteraciones son asintomáticas, dado que la actividad paroxística se traduce clínicamente como actividad irritativa. La misma no se manifestó por historia en ninguno de nuestros casos.

La actividad epileptiforme encontrada, podría estar en relación al déficit de micronutrientes como la piridoxina o tiamina. El déficit de piridoxina es una importante causa de crisis epilépticas en niños, principalmente en recién nacidos y lactantes menores ${ }^{(9-13)}$. También se puede encontrar déficit de piridoxina en los infantes o escolares ${ }^{(11-13)}$. Dicha carencia produce defecto en la adecuada síntesis de $\mathrm{GABA}^{(1-11)}$. Otros mecanismos han sido postulados como los déficits enzimáticos a los cuales se pueden predisponer ${ }^{(13)}$. Ésto manifiesta crisis epilépticas que pueden ser focales, multifocales, al igual que la actividad electroencefalográfica en donde 
se pueden observar puntas y complejos de punta-onda con, incluso, hipsarritmia. Estos hallazgos se omiten al administrar piridoxina intravenosa ${ }^{(14-18)}$.

El déficit de tiamina puede producir cambios electroencefalográficos, sobre todo cuando se acompaña de encefalopatía de Wernicke, se ha descrito presencia de complejos de punta onda ${ }^{(14,15)}$. La pelagra también puede producir alteraciones como: actividades lentas de forma generalizada o focal ${ }^{(17)}$.

No hubo mayor diferencia entre los sexos, sin embargo encontramos que algunos tipos de anormalidades electroencefalográficas fueron más frecuentes en sexo femenino (la actividad lenta focal) y otras en el masculino (actividad paroxística focal y generalizada). Los estudios no describen si un sexo es más propenso a padecer diferentes tipos de alteraciones.

En cuanto a los grupos de edad, parece ser que los más afectados son los menores, encontrándose la mayor frecuencia de hallazgos anormales dentro de los grupos de 6-7 años y 8-9 años, muy probablemente por susceptibilidad a desarrollar desnutrición. Así mismo, hay aumento de las demandas por el crecimiento y desarrollo. Al establecer una comparación con diferentes autores, nosotros encontramos similitudes, dado que la desnutrición es más frecuente en estas edades; siendo más susceptibles a los cambios y alteraciones en el EEG. También es importante mencionar que dichas edades se encuentran todavía madurando su actividad cerebral.

Por último, nuestros resultados presentan diferencias significativas en cuanto a lo diferentes tipos de hallazgos, no así en la frecuencia de la actividad de fondo. Sin embargo, nosotros observamos que la tendencia de las frecuencias de actividad de fondo es mayor en los controles sanos, pues es mayor la diferencia cuando los pacientes presentan DPE moderada. Dicha característica está condicionada según la edad del paciente. Como se describe en la literatura, el ritmo alfa se adquiere en las edades preescolares y se establece o consolida alrededor de los 7 años.

La media de frecuencias esperada en las edades de 6-12 años es de 10cc/por segundo; otras series reportan $9 \mathrm{cc} / \mathrm{seg}$. La media de amplitudes en las regiones posteriores fue de 56 microvoltios $^{(18-21)}$. En comparación a nuestra serie, no se encontraron diferencias con el grupo control en cuanto a frecuencias de la actividad de fondo, pero sí en la diferencias de amplitudes. No podemos relacionar las diferencias de hemoglobina de los grupos con las anormalidades en el EEG, pero sí con la desnutrición. No se han reportado cambios electroencefalográficos en 
relación con anemia. Los reportes publicados son cuando hay deficiencia de vitamina B12 $2^{(14,16,21)}$.

\section{CONCLUSIONES}

Un 42\% de los pacientes con desnutrición proteico-energética mostraron trazados electroencefalográficos anormales ( $P=0.000045)$ : a) Actividad epileptiforme focal en un $20 \%$, b) Actividad lenta focal en un $15 \%$, c) Actividad epileptiforme generalizada, un $12.5 \%$, d) Actividad lenta generalizada en $10 \%$.

Las alteraciones electroencefalográficas que se encontraron se distribuyeron en mayor cantidad dentro de los grupos de menor edad: 6-7 años y 8-9 años. Los tipos de desnutrición asociados a las alteraciones electroencefalográficas fueron por su mayor frecuencia: la desnutrición proteico-energética aguda leve, desnutrición proteico-energética aguda moderada, desnutrición proteico-energética crónica moderada.

En este estudio el sexo no influyó en el tipo de alteraciones electroencefalográficas.

Este es uno de los primeros estudios en Honduras donde se compara con electroencefalograma un grupo de pacientes con DPE y un grupo de control; encontrando alteraciones electroencefalográficas en el grupo de pacientes con desnutrición con significancia estadística.

\section{RECOMENDACIONES}

1. Dado que la desnutrición produce alteraciones electroencefalográficas, se deben proponer nuevos protocolos de investigación con mayores poblaciones; aplicando estudios funcionales, neuropsicológicos y niveles de micronutrientes.

2. Así mismo deben fomentar otro tipo de estudios electrofisiológicos como los potenciales evocados somato sensoriales, velocidad de conducción nerviosa, EMG con el objetivo de evaluar otros niveles del sistema nervioso.

\section{CONTRIBUCIÓN DE LOSAUTORES}

\section{Dr. Miguel Alonso Solano Blanco}

Autor Principal

Participación completa en todos los apartados del presente estudio y responsable por la integridad de los datos, objetividad y certeza de los resultados. 


\section{Dr. Marco Tulio Medina Hernández \\ Co-autor, asesor Principal}

Participación en el diseño del estudio, evaluación clínica y electroencefalográfica de los participantes, análisis e interpretación de los datos, revisión de la redacción del manuscrito, supervisión durante todo el proceso de investigación.

\section{Dra. Rebeca Hernández Toránzo:}

Co-autor, asesora

Participación completa en la evaluación clínica y electroencefalográfica, diseño, análisis de datos e interpretación.

\section{Dr. José Ángel Lara Pinto:}

Co-autor, asesor en análisis estadístico

Participación en el análisis estadístico e interpretación de datos.

\section{AGRADECIMIENTOS}

a) Por el desarrollo del estudio:

A los pacientes y personas sanas que permitieron la realización del estudio electroencefalográfico como apoyo a la ciencia. Las autoridades de la Escuela 15 de Septiembre de Comayagüela, a la Dra María Rivera (médico epidemióloga), Dra. Claudia Amador (Neurología), Dra. Carmen Aguilar, Dra. Sofía Dubón, Dr. Arnold Thompson, Dr. José Medardo Lara, Dra. Vanessa Sirias (Médicos Residentes del Postgrado de Neurología 2007-2008), Bachiller Janio García y Carlos Velásquez, Walter Giron (IV año medicina) Dr Martín Medina (Pediatra Neumólogo), Dr Kento Holden (Neurología pediátrica), Dr Theodore Munsat( Neurología y WFN).

b) Personales: A Dios por permitirnos llegar hasta donde hemos llegado, a mi esposa e hijos, a mis padres y suegros quienes me apoyaron y confiaron en mí.

\section{BIBLIOGRAFÍA}

Fay S. Tyner, Knott, Mayer Bremm. Fundamentals of EEG Technology Vol 1, 1984, 1-9, 182-267.

Food and Agriculture Organization of the United Nations. Undernourishment around the world. In: The State of Food Insecurity in the World 2004. Rome: FAO, 2004: 10-25.

Frantzen E. Wernicke encephalopathy, Acta. Neurological Scandinava, 1966:42.426-441. 
Goutiere F, Aicardi j, Atypical presentation of pyridoxine dependent seizures; a tractable cause of intractable epilepsy in infants. Annales of neurology, 1985: 17: 117-12.

Hernández R, Hesse H. Rodríguez LC, Medina MT, Neurophysiologic Findings in Malnutrition, In: Medina MT. Seminars in Clinical Neurology: Neurologic Consequences of Malnutrition. First ed.New York: Demos Medical Publishing. 2007:57-65.

Hu WT, Murray JA, Greenaway MC, Parisi JE, Josephs KA Cognitive impairment and celiac disease. Arch Neurol. 2006;63(10):1440-6.

Hunt A. D., Stokes J., McCrory W., et al Pyridoxine depending, Report of case of intractable convulsions in an infant controlled by pyridoxine. Pediatrics, 1954:13:140-145.

Jeffery D. Nutrition and diseases of the nervous system. In: Modern Nutrition in Health and Disease. 9th ed. Philadelphia: Lippincott Williams \& Wilkins, 1999: 80-115.

Kitaoka K, HattoriA, Chikahisa S, Miyamoto K, Nakaya Y, Sei H. Vitamin A deficiency induces a decrease in EEG delta power during sleep in mice. Brain Res. 2007 30;1150:121-30.

Leonberg, S.C., Magnament of infants and children for electroencephalography. Clinical electroencephalography,1987, 15: 202.207.

Needlman R, crecimiento y desarrollo, en: Berhman R, Kliegman $R$, Jenson $H$, tratado de pediatría Nelson, 16 ed. Mexico DF: McGraw-Hill, 2001: $\quad 62-6$

Nelson G. K., The electroencephalogram in Kwashiorkor. Electroencephalogramclinical neurophysiology.1959: 11:73-84.

Niedermeyer E, Maturation of the EEG, In: Niedermeyer E., Lopez Dasilva, F.,Electroencephalography, 5ed, , Philadelphia, Lippincott Williams, 2005, 11:208-232.

Petersen I. Eeg-Olofsson, O. the development of the electroencephalogram in normal children from the age 1-15years, Neuropediatrie,1990 2:247-302.

Rodríguez LC, Amador C, Medina MT, Malnutrition and Neurologic Disorders: A Global Overview, In: Medina MT. Seminars in Clinical Neurology: Neurologic Consequences of Malnutrition. First ed, New York, Demos Medical Publishing. 2007:1-19

Ropper A, Brown R. Diseases of the nervous system due to nutritional deficiency. In: Brown R. Adams and Victor's Principles of Neurology. 8th ed. New York: McGraw-Hill, 2005;983-1003.

Rudman D, Williams P. j, Megadoses of vitamins, use and misuse, New England Journal of Medicine, 1983: 309:488-490.

Srikantia S, Veeraraghava Reddy, Krishnaswamy K. Electroencephalographic patterns in pellagra. Electroencephalography clinical neurophysiology.1968: 
25:386-388.

Vas G, Cracco J, diffuse encephalopaties, In: Daly D, Pedley T. Current practice of clinical electroencepholography. 2 ed. New York: Raven press 1990: 2: 385386.

Waterlow JC, classification and definition of protein calorie malnutrition. malnutrition,1972; 5(66): 12-30.

Waterlow JC, Buzina R, Keller W, Lane JM, Nichaman MZ, Tanner JM. The presentation and use of height and weight data for comparing the nutritional status of groups of children under the age of 10 years. Bulletin of the World Health Organization 1977; 55: 489-498. 